\title{
Die Liposuktion mit Vibrationsmikrokanülen ist eine erfolgreiche Therapie des Lipödems
}

\section{Liposuction with vibrating microcannulas is an effective treatment for lipedema}

\author{
Autoren \\ Kristina Korsake', Stefan Rapprich ${ }^{2}$ \\ Institute \\ 1 Ambulantes OP-Zentrum und Venen-Kompetenzzentrum \\ Bodensee, Radolfzell; \\ 2 Hautmedizin Bad Soden, Bad Soden am Taunus \\ Schlüsselwörter \\ Liposuktion, Lipödem, Lipolymphödem, Lipohyperthrophie, \\ Chirurgie \\ Key words \\ lipedema, lipolymphedema, lipohypertrophy, liposuction, \\ surgery

\section{Bibliografie} \\ DOI https://doi.org/10.1055/a-1155-3503 \\ Online-Publikation: 14.5.2020 \\ Phlebologie 2020; 49: 171-177 \\ (c) Georg Thieme Verlag KG, Stuttgart · New York \\ ISSN 0939-978X \\ Korrespondenzadresse \\ Dr. med. Kristina Korsake \\ Ambulantes OP-Zentrum und Venen-Kompetenzzentrum \\ Bodensee, Walchnerstr. 16, 78315 Radolfzell \\ kristina.korsake@gmail.com
}

\section{ZUSAMMENFASSUNG}

Das Lipödem ist eine chronische, progrediente, schmerzhafte, übermäßige Fettgewebsvermehrung bei Frauen mit deutlicher Disproportion zwischen Stamm und Extremitäten [1]. Zusätzlich bestehen Ödeme, die durch Orthostase verstärkt werden, sowie eine Hämatomneigung nach Bagatelltraumen [2-4]. Die Ursache ist unbekannt, daher gibt es keine kausale Therapiemöglichkeit. Die vielfach empfohlene komplexe physikalische Entstauungstherapie (KPE), bestehend aus manueller Lymphdrainage und Kompression, ist nur gegen die Ödemkomponente wirksam. Eine dauerhafte Reduktion des krankhaften Unterhautfettgewebes an Beinen und Armen ist mittels Liposuktion durch die Mikrokanülentechnik in Tumeszenz-Lokalanästhesie möglich geworden [5].

\section{ABSTRACT}

Lipedema is a chronic, progressive, painful, increased deposition subcutaneous fat tissue in women with a clear disproportion between the trunk and extremities. Lipedema offen lead to oedema, which are worsened by orthostasis, and hematoma after minor injury. The pathogenesis is unknown and no curative treatment is available. Conservative therapy consisting of lymphatic drainage and compression stockings is often recommended, but is only effective against the edema component. Some patients show a short-term improvement when treated in this way. Permanent reduction of the pathological subcutaneous fat on the legs and arms has become possible by employing advanced liposuction techniques using microcannula technology in local tumescent anaesthesia.

\section{Definition}

Beim Lipödem handelt es sich um eine chronische, progrediente Erkrankung, die durch eine übermäßige Fettgewebsvermehrung der Extremitäten bei Frauen gekennzeichnet ist [1]. Charakteristisch sind Berührungs-, Druck- und Spannungsschmerzen, Ödeme, die durch Orthostase verstärkt werden, sowie eine Hämatomneigung nach Bagatelltraumen [2-4].

Die Daten der ambulant durchgeführten Studien zeigen eine Häufigkeit der Erkrankung von 7-9,7 \% [6-8]. Insgesamt geht man angesichts der weitverbreiteten Unsicherheit bezüglich der Diagnosestellung von einer hohen Dunkelziffer aus [9]. Die Ursache des Lipödems ist unbekannt. Eine Rolle in der Pathogenese spielen sicher hormonelle Aspekte, da das Lipödem nahezu ausschließlich bei Frauen auftritt und in der Regel in einer Phase hormoneller Veränderungen wie Pubertät, Schwangerschaft, Hormoneinnahme oder Klimakterium beginnt oder manifestiert [2]. Der Progress ist nicht vorhersehbar und individuell unterschiedlich. Außer den hormonellen Aspekten ist von einer genetischen 


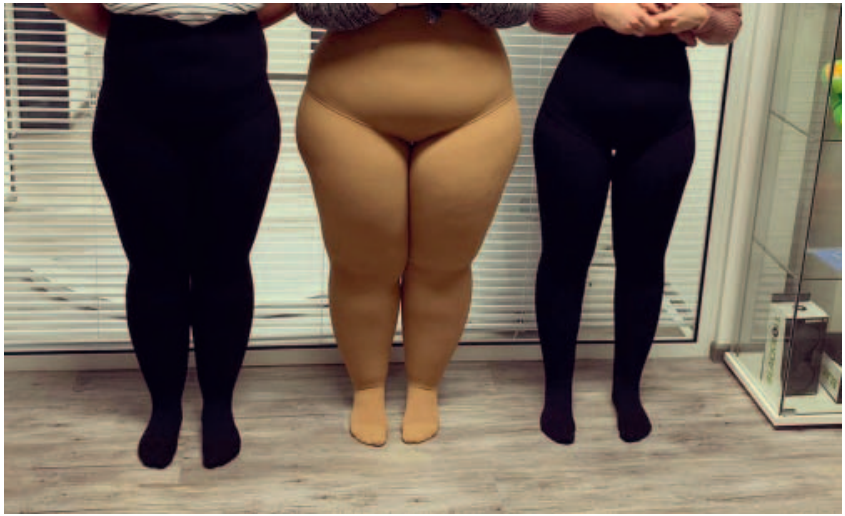

- Abb. 1 Die 3 Schwestern leiden am Lipödem.

Disposition auszugehen, da häufig mehrere weibliche Mitglieder einer Familie betroffen sind ( $\boldsymbol{A} \mathbf{A b b} \mathbf{1} \mathbf{1})$.

Pathophysiologisch bedeutsam ist eine nachgewiesene erhöhte Kapillarpermeabilität, die zu orthostatischen Ödemen führt. Diese sind für die erhöhte Berührungs- und Druckempfindlichkeit des Gewebes verantwortlich, nicht die Fettmenge. Eine gleichzeitig bestehende erhöhte Kapillarfragilität erklärt die bei allen Patientinnen bestehende Hämatomneigung.

\section{Diagnosestellung}

Die Diagnosestellung erfolgt klinisch, wobei das Lipödem insbesondere von der Lipohypertrophie und dem Lymphödem abzugrenzen ist. Man unterscheidet einen Ganzbein-, Oberschenkelund Unterschenkel-Typ. In etwa 30\% der Fälle sind auch die Arme betroffen [4] ( Abb. 2, 3). Die Stadieneinteilungen des Lipödems sind mit dem morphologischen Bild und nicht zwangsläufig mit dem Ausmaß der klinischen Schmerzsymptomatik verknüpft $(\triangleright$ Tab. 1).

Sonografisch kann eine homogene Verbreiterung der Subkutis mit gleichmäßig vermehrter Echogenität und echoreichen Septen dargestellt werden [10].

\section{Therapie}

Ziel der Therapie ist die Beseitigung oder Besserung der Beschwerden (Schmerzen, Ödem und Disproportion) sowie die Verhinderung von dermatologischen, lymphatischen und orthopädischen Komplikationen. Eine kausale Therapie ist nicht bekannt. Die kombinierte physikalische Entstauungstherapie (KPE) ist gegen die Ödemkomponente wirksam. Lediglich ein Teil der Patientinnen erfährt dadurch eine Besserung der Beschwerden $[4,11,12]$. KPE beinhaltet Kompressionstherapie, manuelle Lymphdrainage, Bewegungstherapie und Hautpflege.

\section{Liposuktion}

Zur dauerhaften Reduktion des krankhaften Unterhautfettgewebes an Extremitäten und zur Minderung der Leidenssituation der be-

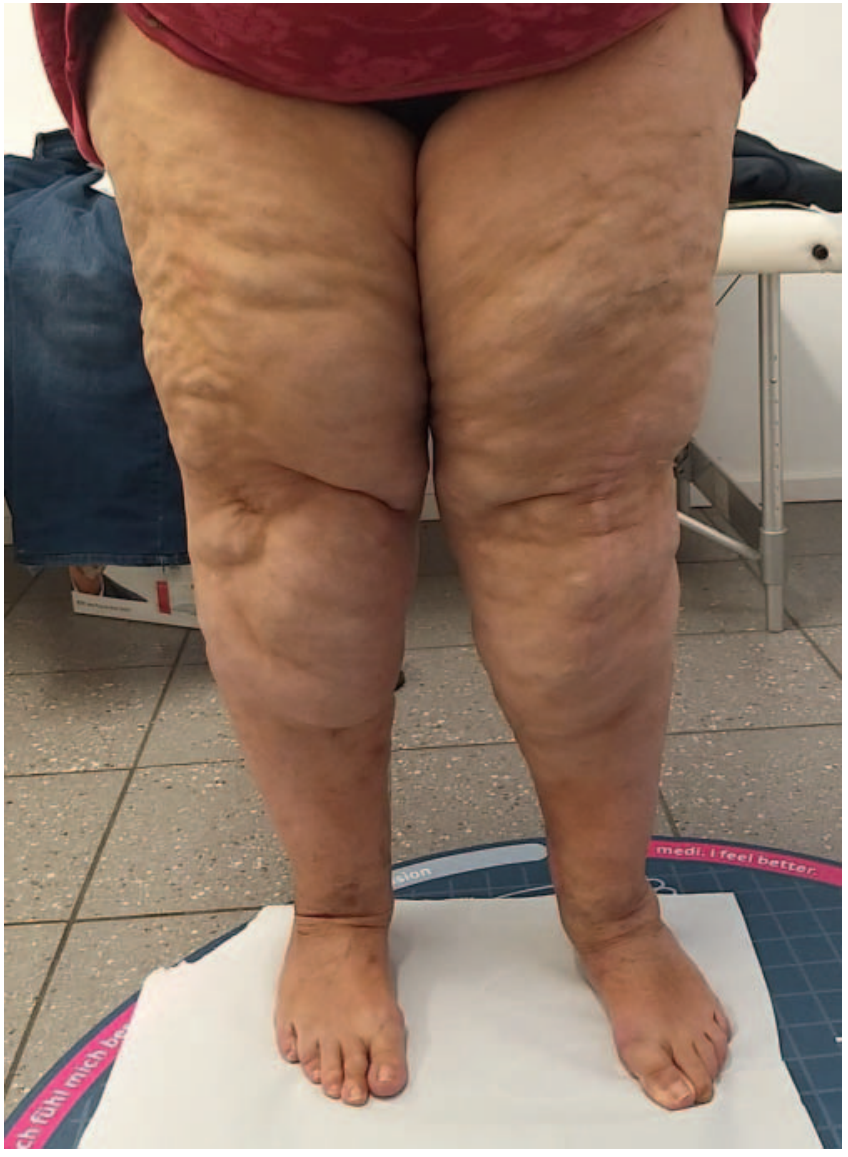

- Abb.2 Ausgeprägte Umfangsvermehrung, grobe, deformierende Fettlappen an den Innenseiten der Oberschenkel bei Lipödem Stadium III, häufig findet sich das subpatellare Fettpolster.

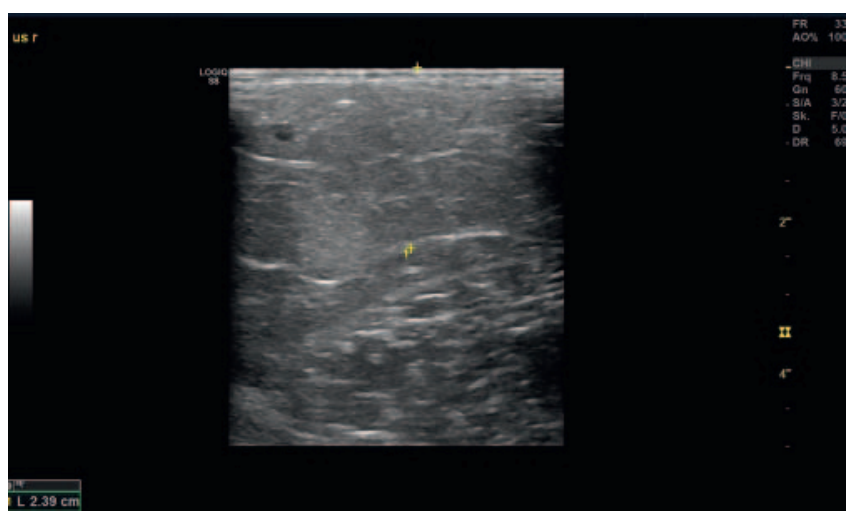

- Abb. 3 Sonomorphologische Darstellung des Lipödems anhand der gemeinsamen Dicke von Kutis und Subkutis 6-8 cm oberhalb des Malleolus medialis.

troffenen Patientinnen wird die Liposuktion eingesetzt. Sie ist insbesondere dann angezeigt, wenn trotz konsequent durchgeführter konservativer Therapie noch Beschwerden bestehen [5, 13].

Der Eingriff kann ambulant oder stationär erfolgen [5, 14, 15]. Die Indikation und der Therapieplan müssen - unter Berücksichtigung von Patientenfaktoren - jeweils individuell gestellt wer- 
- Tab. 1 Einteilung des Lipödems nach Morphologie.

\begin{tabular}{|c|l|}
\hline Stadium & Charakteristik \\
\hline I & $\begin{array}{l}\text { Subkutis gleichmäßig verdickt und weich mit kleinen } \\
\text { Knötchen, Haut glatt }\end{array}$ \\
\hline II & $\begin{array}{l}\text { Subkutis verdickt und weich mit größeren Knoten, } \\
\text { unebene wellenartige Hautoberfläche }\end{array}$ \\
\hline III & $\begin{array}{l}\text { Subkutis verdickt und induriert, große Knoten, Wammen- } \\
\text { artige, deformierende Fettlappen an den Innenseiten von } \\
\text { Oberschenkeln und Knien }\end{array}$ \\
\hline
\end{tabular}

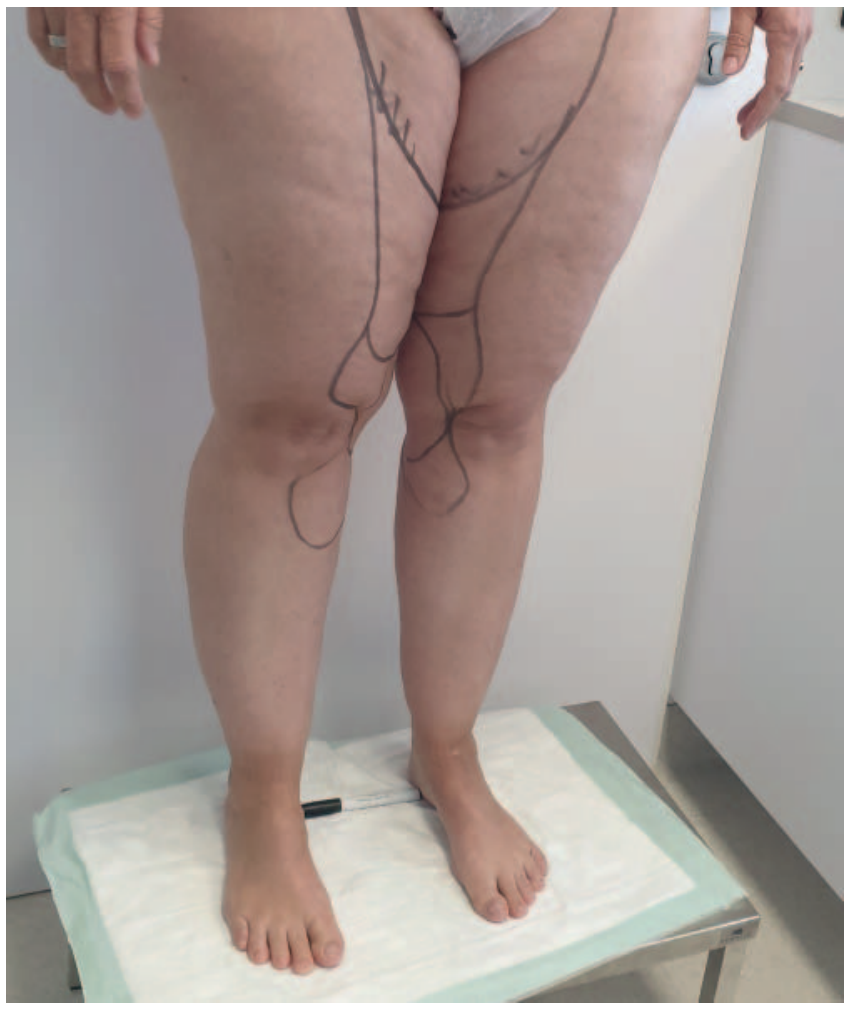

- Abb.4 Markierung der Behandlungsareale an der Oberschenkelinnenseite vor Liposuktion.

den. Für die Liposuktion beim Lipödem der unteren Extremitäten kommen folgende Areale in Betracht: Oberschenkelinnenseite, Oberschenkelaußenseite, Unterschenkel ( $\mathbf{A b b . 4 , 5 ) . ~ Z w i s c h e n ~}$ der Absaugung zweier Areale soll ein zeitlicher Abstand von mindestens 4-6 Wochen liegen.

Seit der Einführung der Tumeszenz-Lokalanästhesie (TLA) Ende der 1980er-Jahre wird die „trockene“ Liposuktion aufgrund möglicher Lymphgefäßverletzungen nicht mehr angewendet [16]. Die Liposuktion sollte in örtlicher Betäubung mittels TLA und mit stumpfen Mikrokanülen durchgeführt werden [14, 17-21] ( $\triangleright$ Abb. 6). Der erfahrene Operateur kann in TLA die Körperkonturen mit zunehmender Entleerung des Lipödems realistisch beurteilen und ggf. sofort Korrekturen vornehmen. Die Liposuktion

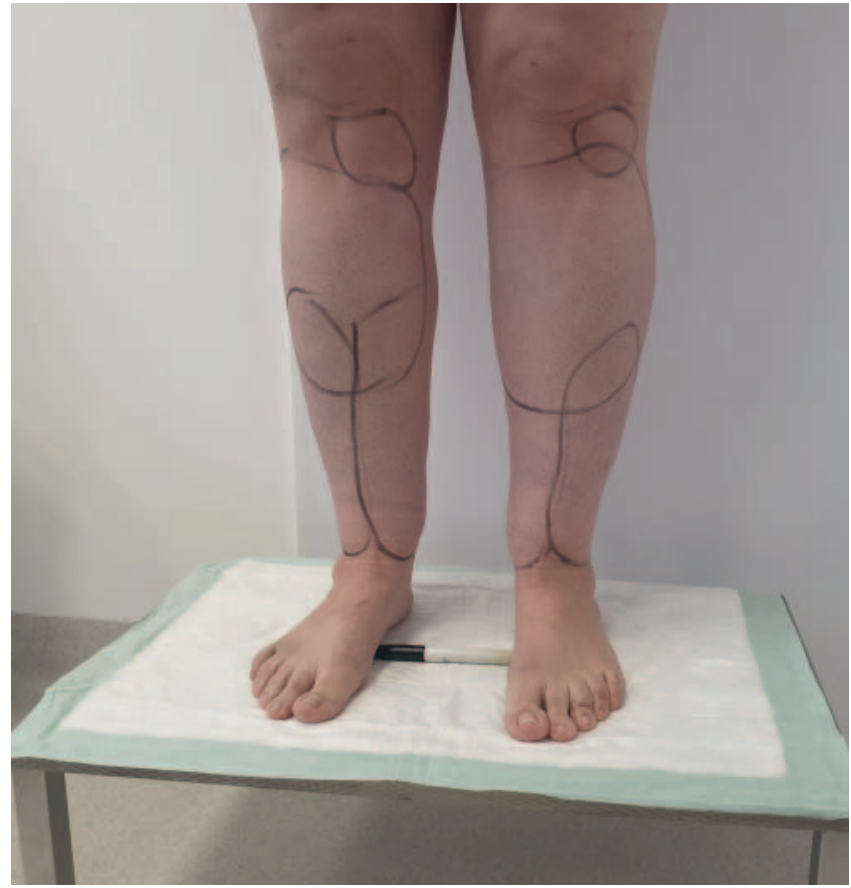

- Abb. 5 Markierung der Behandlungsareale am Unterschenkel vor Liposuktion.

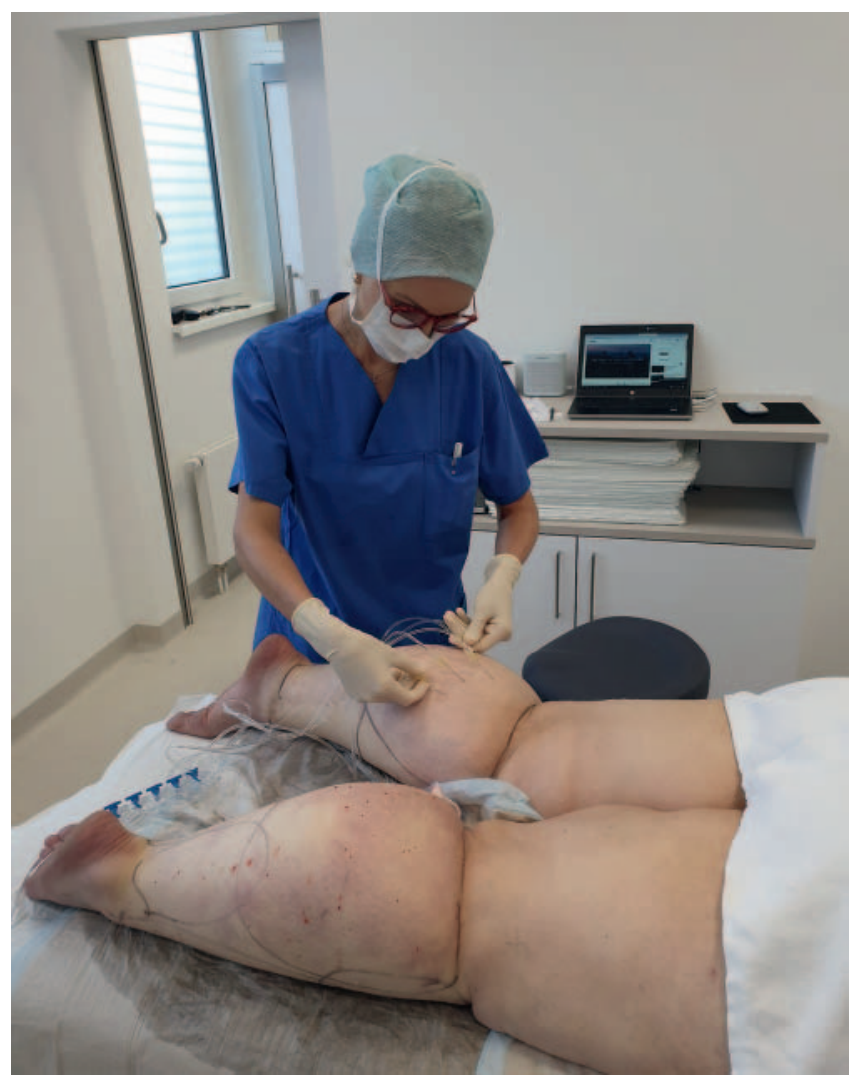

- Abb. 6 Setzen der Tumeszenz-Lokalanästhesie. 


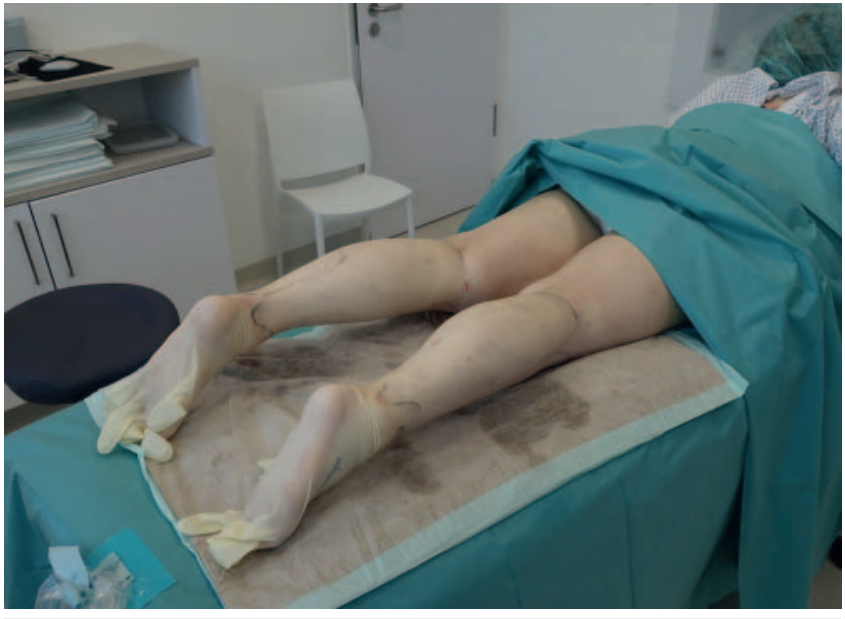

- Abb. 7 Lagerung auf dem OP-Tisch.

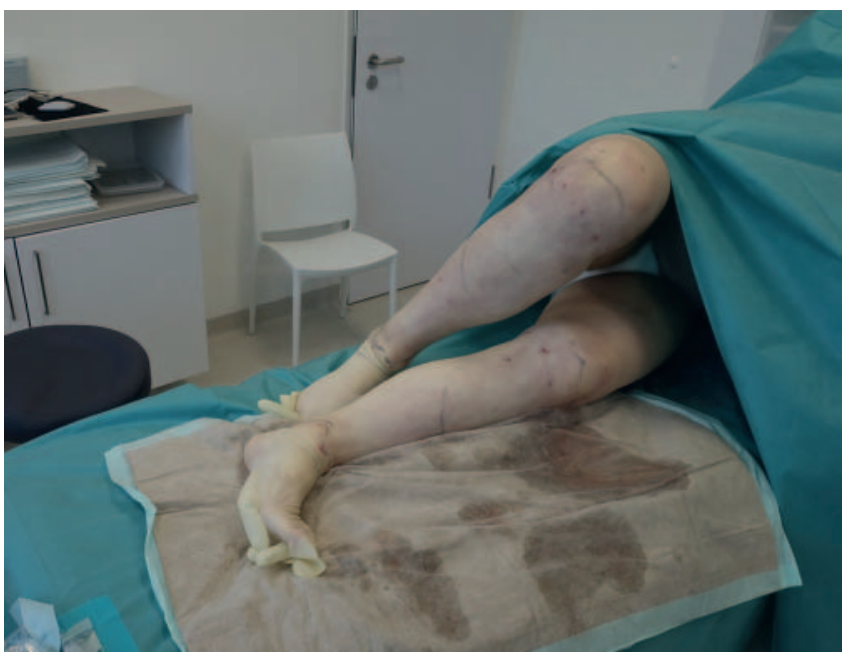

- Abb. 8 Patientin hat ihre Lage selbstständig verändert.

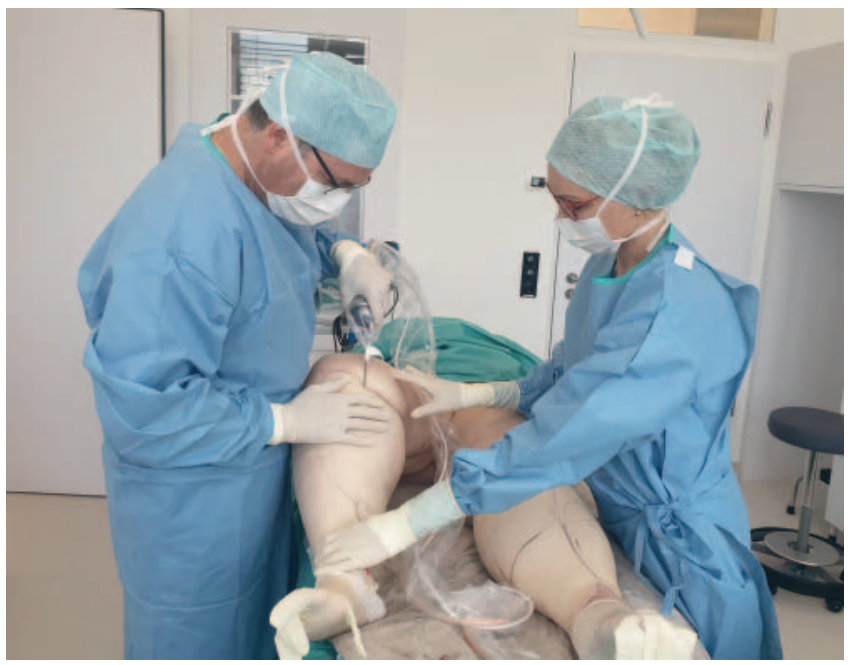

- Abb.9 Liposuktion mithilfe einer stumpfen Vibrationskanüle.

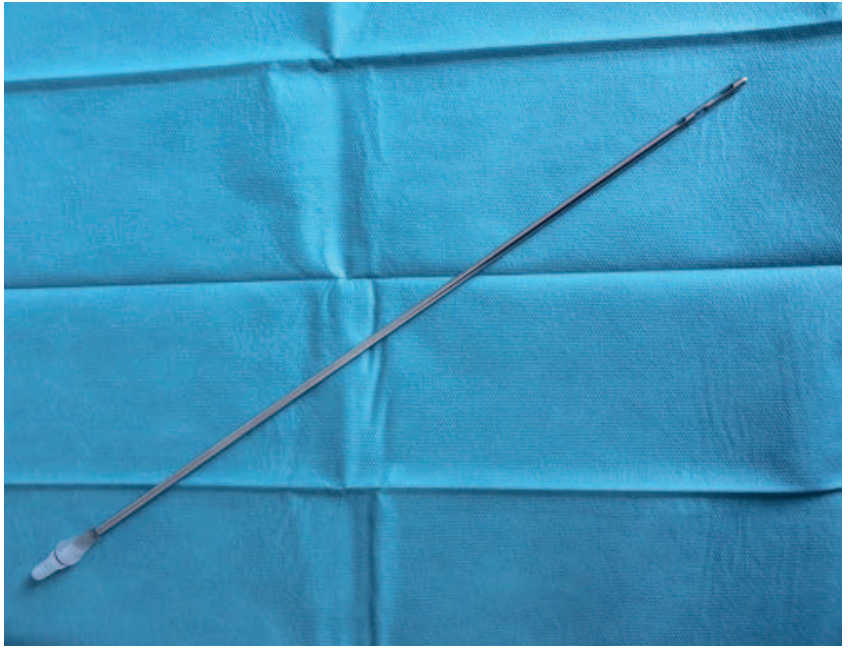

- Abb.10 Kanüle - heute kommen hauptsächlich vibrierende Kanülen mit 2-5 mm Durchmesser, mit einem stumpfen Ende und verschiedenen Ausführungen zum Einsatz.

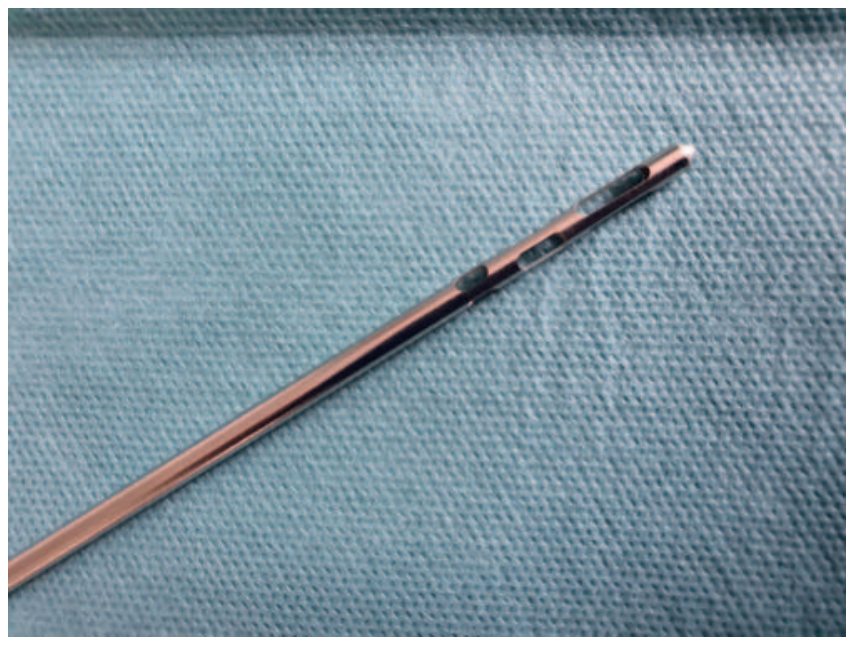

- Abb. 11 Die Autoren bevorzugen die 4 mm-Kanüle.

wird mittels gewebeschonenden Techniken wie Vibration oder Wasserstrahl mit stumpfen Mikrokanülen durchgeführt [22] ( Abb. 7-14).

Die maximale Gesamtdosis von Lidocain liegt bei $55 \mathrm{mg} / \mathrm{kg}$ bei Frauen $<60$ Jahren und bei $45 \mathrm{mg} / \mathrm{kg}$ bei Frauen über 60 Jahren [21].

Der Eingriff führt zu ausgeprägten Verbesserungen von Spontanschmerz, Druckschmerz, Ödem und Hämatomneigung mit signifikanten Unterschieden prä- und postoperativ $[5,14,19]$ ( $\sim$ Abb. 15). Es wird eine Verminderung der konservativen Therapie, z. T. sogar eine Therapiefreiheit erzielt [5, 14, 15]. Die Befundbesserungen bleiben mehrheitlich über viele Jahre bestehen [ 5 , 14, 23]. Weiterhin werden durch die Reduktion der Fettgewebsdepots an den Oberschenkel- und Knieinnenseiten die mechanisch und okklusiv bedingten Hautschäden reduziert bzw. beseitigt. Die Korrektur der Beinfehlstellung bewirkt eine Besserung der Beweglichkeit und des Gangbildes [24] sowie eine Reduktion 


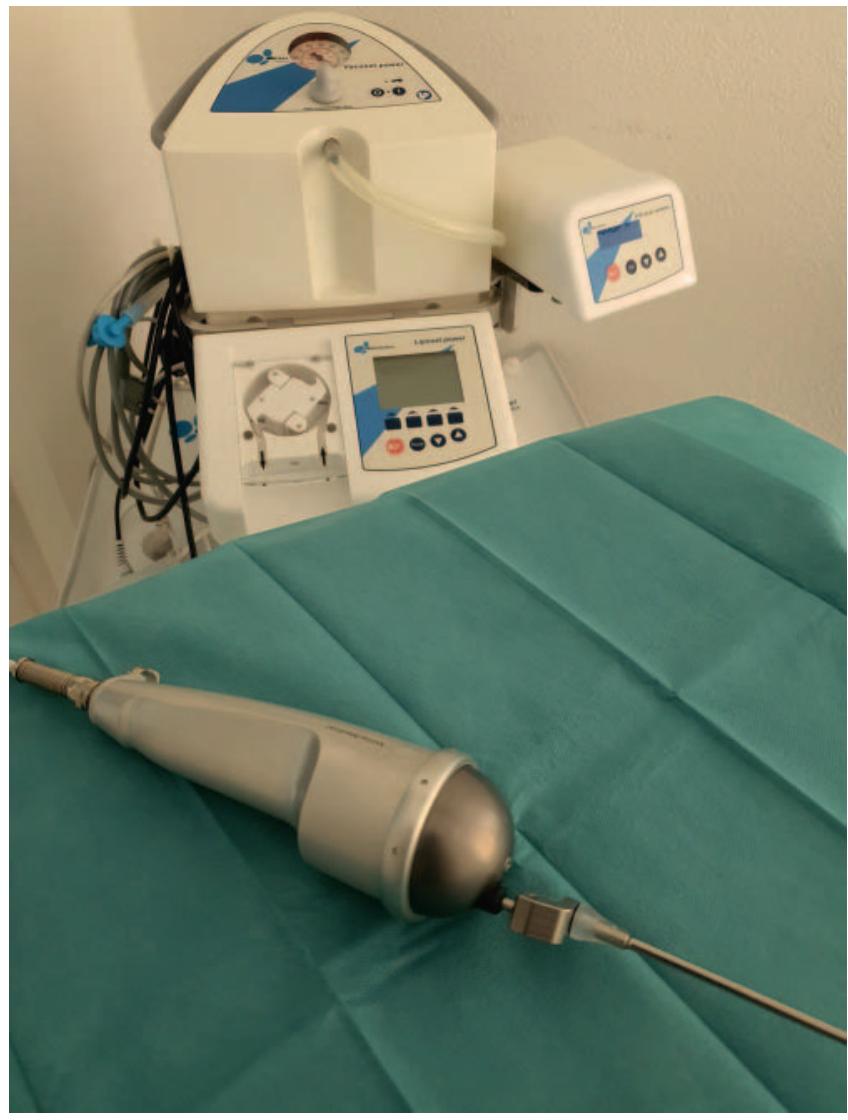

- Abb.12 Ein Motor im Handstück versetzt die Absaugsonde in Längsrichtung in eine Vibration von 4000 bis 5000 Schwingungen pro Minute.

des Risikos für weitere orthopädische Komplikationen infolge des Lipödem-assoziierten pathologischen Gangbildes (z. B. Gon- und auch Coxarthrosen).

Durch die Beschwerdeminderung, die vermehrte Mobilität, den geringeren Zeitaufwand für die konservative Therapie und das wieder gesteigerte Selbstbewusstsein bessert sich die Lebensqualität der Betroffenen deutlich. In der Liposuktion erfahrene Operateure raten zu einer kritischen Indikationsstellung bei einem Körpergewicht $>120 \mathrm{~kg}$ oder einem BMI $>32 \mathrm{~kg} / \mathrm{m} 2$ [13]. Eine begleitend zum Lipödem bestehende morbide Adipositas sollte vor einer Liposuktion therapeutisch angegangen werden. Letztlich liegt die Indikationsstellung bzw. Durchführung der Liposuktion im Ermessen des Operateurs. Die Liposuktion ist keine Methode zur Gewichtsreduktion [13]. Nach einer umfassenden Fettabsaugung bei ausgeprägtem Lipödem kann das resultierende überschüssige Hautgewebe die langfristige Beschwerdereduktion beeinträchtigen. Die Daten der klinischen Studien legen nahe, dass die Fettabsaugung bei Lipödemen im Stadium I und II die Beeinträchtigung der Lebensqualität nachhaltiger verringert und den Bedarf an konservativer Therapie stärker verringert als die Fettabsaugung bei Lipödemen im Stadium III [14, 25] ( $\triangleright$ Abb. 16).

Die früh postoperative Nachbehandlung bei Liposuktion mittels KPE wird solange durchgeführt, wie eine Schwellung nachweisbar ist (Dellbarkeit). Im Stadium I benötigen fast alle Patientinnen nur 3-6 Wochen eine postoperative Nachbehandlung,

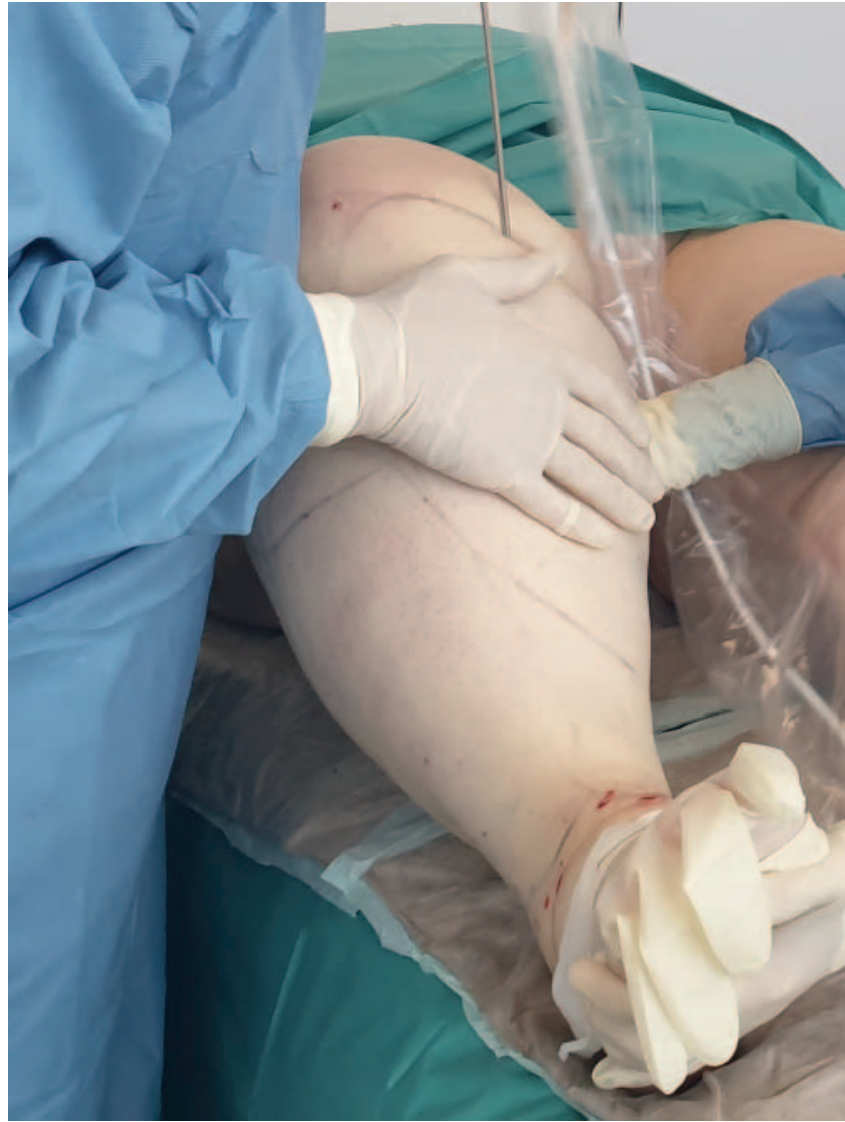

Abb. 13 Der Operateur bewegt mit 1 Hand die Kanüle in verschiedene Richtungen fächerförmig vor und zurück, um die Fettdepots möglichst gleichmäßig zu reduzieren.

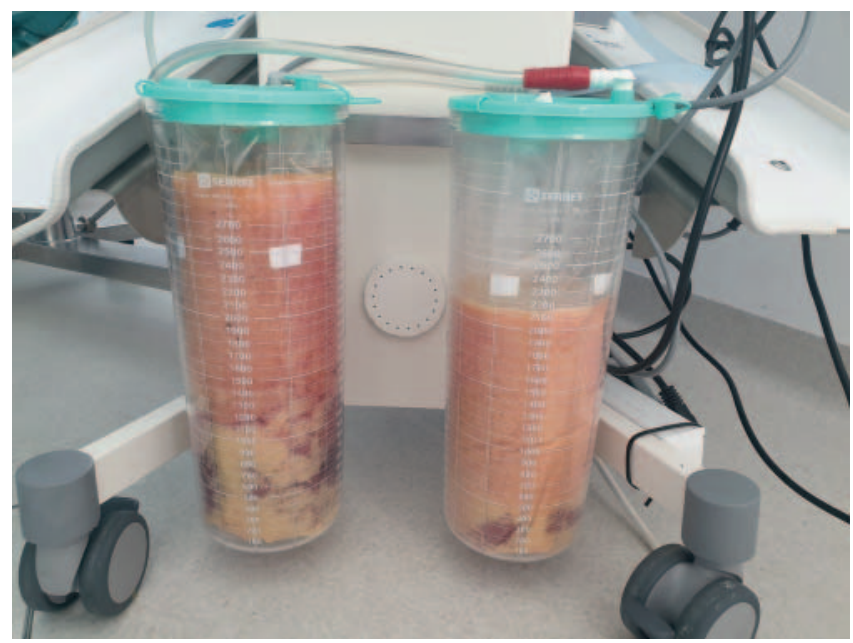

- Abb.14 Das Aspirat setzt sich aus dem oben schwimmenden Fett und der darunter liegenden Mischung aus Tumeszenzlösung und Blutspuren zusammen. Gelbes Aspirat ist ein Indikator für geringen Blutanteil und die atraumatische Methode.

dann nicht mehr. Je weiter fortgeschritten wird die postoperative KPE länger benötigt, manchmal auf Dauer, dann aber oft in geringerer Intensität und Frequenz [5, 8, 21, 26]. 


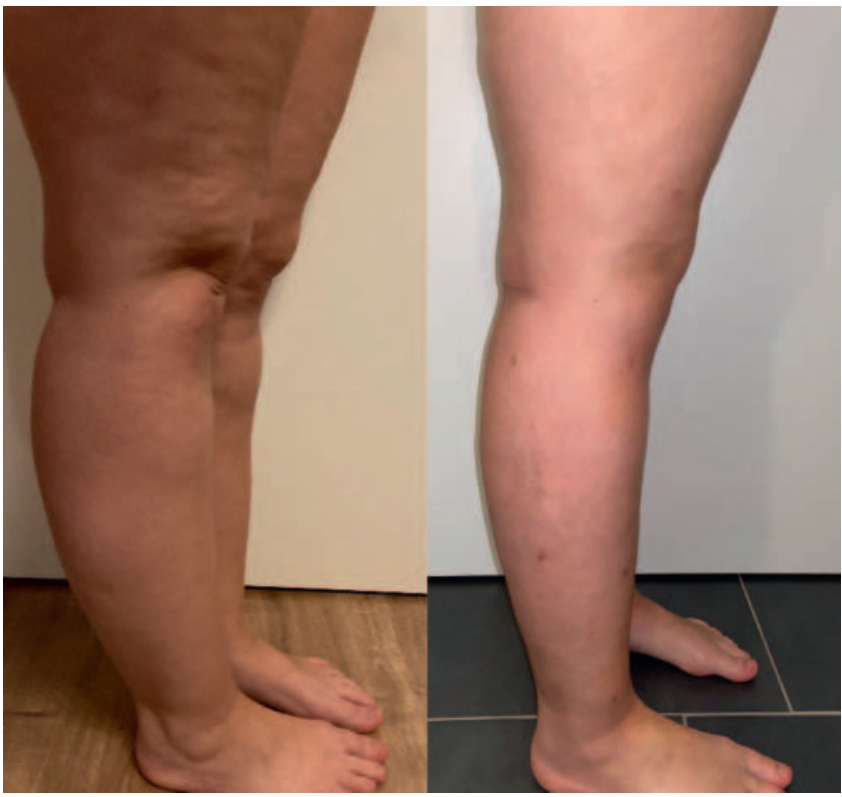

- Abb. 15 Lipödem-Patientin 2 Tage und 20 Tage nach Liposuktion.

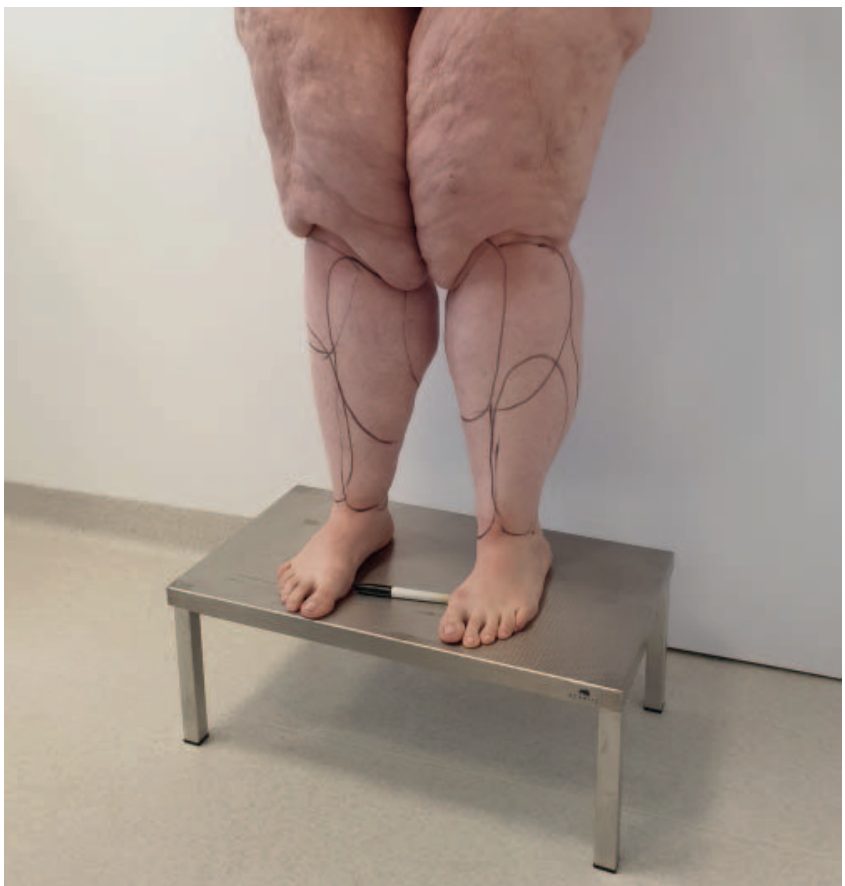

- Abb.16 Überschüssiges Hautgewebe am Oberschenkel bei Z.n. Liposuktion bei Lipödem Stadium III.

Die Studiendaten zeigen, dass die Liposuktion eine wirksame Behandlung für Lipödeme mit guten Langzeitergebnissen ist, zu einer signifikanten Verringerung des Bedarfs an konservativer Therapie führt und auch die Lebensqualität dieser Patientinnen deutlich verbessert [14, 25-27]. Das Risiko einer Komplikation im Rahmen einer Liposuktion kann als sehr gering bezeichnet werden, mit $<5 \%$ der gesamten unerwünschten Ereignisse [14, 25-28] und postoperativen Infektionsraten von 1-2\% [5, 25] sowie einzelnen Thrombosefällen [14].

\section{Zusammenfassung}

Die Liposuktion beim Lipödem ist bisher die einzige Therapie, die es ermöglicht, langfristig Beschwerden zu lindern, die Lebensqualität deutlich zu verbessern und den Bedarf an konservativer Therapie stärker zu reduzieren, weil sie nicht nur das Ödem, sondern auch das pathologisch veränderte Fettgewebe reduziert. Da es sich um ein chronisches Krankheitsbild handelt, ist der Verlauf - ausgehend vom verbleibenden Fettgewebe - möglicherweise weiter progredient.

Die Ergebnisse sind in Frühstadien bei jungen Patientinnen besser als bei ausgeprägten Befunden in höherem Alter. Die präund postoperative KPE hat im Rahmen einer Therapiekombination einen hohen Stellenwert.

Die Qualität der aktuell verfügbaren Evidenz ist begrenzt. Es fehlen randomisierte und kontrollierte Studien. Weitere Validierungen der Ergebnisse und Definition eines minimal klinisch wichtigen Unterschieds für die Erkrankung können ebenfalls erforderlich sein, um den Nutzen der Fettabsaugung für die Behandlung von Lipödemen in eine klinische Perspektive zu rücken.

Interessenkonflikt

Die Autorinnen/Autoren geben an, dass kein Interessenkonflikt besteht.

Literatur

[1] Herpertz U. Krankheitsspektrum des Lipödems an einer Lymphologischen Fachklinik - Erscheinungsformen, Mischbilder und Behandlungsmöglichkeiten. Vasomed 1997; 5: 301-330

[2] Allen EV, Hines EA. Lipedema of the legs. Proc Mayo Clin 1940; 15 : 184-187

[3] Wienert V, Leeman S. Das Lipödem. Hautarzt 1991; 42: 484-486

[4] Herpertz U. Ödeme und Lymphdrainage. Diagnose und Therapie. In: Herpertz U, edt Lehrbuch der Ödematologie. 5. Aufl Stuttgart: Schattauer; 2014: 41

[5] Schmeller W, Hueppe M, Meier-Vollrath I. Liposuction in lipoedema yields good long-term results. Brit J Dermatol 2012; 166: 161-168. doi:10.1111/j.1365-2133.2011.10566.x

[6] Miller A. Komorbidität von Patienten mit Lymphödemen. LymphForsch 2008; $12: 14-18$

[7] Marshall M, Schwahn-Schreiber C. Prävalenz des Lipödems bei berufstätigen Frauen in Deutschland. Phlebologie 2011; 40: 127-134

[8] Rapprich S, Baum S, Kaak I et al. Treatment of lipoedema using liposuction: results of our own surveys. Phlebologie 2015; 44: 121-132

[9] AWMF: S1-Leitlinie 037/012: Lipödem, aktueller Stand 10/2015.

[10] Marshall M, Schwahn-Schreiber C. Lymph-, Lip - und Phlebödem. Differenzialdiagnostische Abklärung mittels hochauflösender Duplexsonografie. Gefäßchirurgie 2008; 3: 204-212

[11] Meier-Vollrath I, Schneider W. Lipödem: Verbesserte Lebensqualität durch Therapiekombination. Deutsches Ärzteblatt 2005; 102: 10611067

[12] Szolnoky G, Varga E, Varga M et al. Lymphedema treatment decreases pain intensity in lipedema. Lymphology 2011; 44: 178-182

[13] Schmeller W, Baumgartner A. Operative Aspekte bei Liposuktion des Lipödems: Zwölf Fragen - zwölf Antworten. LymphForsch 2014; 18 : $6-12$ 
[14] Rapprich S, Dingler A, Podda M. Liposuktion ist eine wirksame Therapie beim Lipödem - Ergebnisse einer Untersuchung mit 25 Patientinnen. J Deutsch Dermatol Ges 2011; 9: 33-40

[15] Cornely ME. Liposuction. Principles and Practice. In: Shiffman MA, Di Giuseppe A, eds; Lipedema and lymphatic edema. Berlin: Springer; 2006: 547-555

[16] Hoffmann JN, Fertmann JP, Baumeister RG et al. Tumescent and dry liposuction of lower extremities: differences in lymph vessel injury. Plast Reconstr Surg 2004; 113: 718-724

[17] Sattler G. Liposuction in lipoedema. Ann Dermatol Venereol 2002; 129: $1 \mathrm{~S} 103$

[18] Cornely ME. Fatter through lipids or water: Lipohyperplasia dolorosa versus lymphedema. American Journal of Cosmetic Surgery 2014; 31: 189-195. doi:10.5992/AJCS-D-12-00026.1

[19] Schmeller W, Meier-Vollrath I. Das Lipödem: neue Möglichkeiten der Therapie. Schweiz Med Forum 2007; 7: 150-155. doi:10.4414/ smf.2007.06113

[20] Rapprich S, Loehnert M, Hagedorn M. Therapy of lipoedema syndrome by liposuction under tumescent local anaesthesia. Ann Dermatol Venerol 2002; 129: 1 S 711

[21] Sandhofer M, Hanke W, Habbema L et al. Prevention of Progression of Lipedema With Liposuction Using Tumescent Local Anesthesia: Results of an International Consensus Conference. Dermatol Surg 2020; 46: $220-228$

[22] Stutz J], Krahl D. Water jet-assisted liposuction for patients with lipoedema: histologic and immunohistologic analysis of the aspirates of 30 lipoedema patients. Aesth Plast Surg 2009; 33: 153-162

[23] Baumgartner A. Operative Therapie - Notwendigkeit oder Luxus? Vasomed 2014; 26: 238

[24] Stutz J. Liposuktion beim Lipödem zur Verhinderung von Gelenkspätkomplikationen. Vasomed 2011; 23: 62-66

[25] Dadras M, Mallinger PJ, Corterier CC et al. Liposuction in the treatment of lipedema: a longitudinal study. Arch Plast Surg 2017; 44: 324-331

[26] Baumgartner A, Hueppe M, Schmeller W. Long-term benefit of liposuction in patients with lipoedema: a follow-up study after an average of 4 and 8 years. Br J Dermatol 2016; 174: 1061-1067

[27] Peprah K, MacDougall D. Liposuction for the Treatment of Lipedema: A Review of Clinical Effectiveness and Guidelines. Ottawa: CADTH; 2019: 06

[28] Habbema L. Safety of liposuction using exclusively tumescent local anesthesia in 3,240 consecutive cases. Dermatol Surg 2009; 35: 17281735 